\title{
Radio virtual comunitaria: Un espacio para mejorar los procesos educativos con adultos mayores ${ }^{1 .}$
}

\author{
Virtual community radio: A space to improve educational processes with older \\ adults.
}

\section{Juan Manuel Montoya ${ }^{2}$ Marlon Echavarría Rodríguez ${ }^{3}$}

Montoya. Juan M, Echavarría R. Marlon. miradas Nº13 - 2015. ISSN: 0122 994X Págs 88 - 99

Recepción: Mayo 15 de 2015

Aprobación: Octubre 9 de 2015

Publicación: Diciembre 17 de 2015

\section{Resumen}

Un mundo globalizado en donde el uso de las tecnologías de la información y comunicación ha llevado a la creación, construcción y consolidación de nuevas propuestas de enseñanza $\mathrm{y}$ aprendizaje. Medios masivos de comunicación que han sido obligados a transformar y evolucionar, para no quedar extintos en el pasado.

Por tal razón, a partir de la interconectividad que ofrece la virtualidad, y de la complejidad que poseen los inmigrantes digitales en el uso de las tecnologías, surge la propuesta de analizar la radio virtual comunitaria como proceso educativo, participativo, comunicativo y de transformación en los adultos mayores de la comuna 11 de la ciudad de Medellín.

La búsqueda de elementos característicos en la comunidad beneficiada del proyecto radial y comunitario, permitió desarrollar una investigación cualitativa en donde por medio del cuestionario, el análisis de información y las entrevistas semiestructuradas se llevó a cabo la investigación "Propuesta para mejorar los procesos educativos con los adultos mayores a través de la radio virtual comunitaria. Caso: Comuna 11 del municipio de Medellín, Antioquia” (Montoya \& Echavarría Rodríguez, 2015)

Dicho proceso permitió determinar que a partir de la creación de audiencia, sigue una transformación social, dado que el contenido que se emite en los programas de La Once Radio genera un aprendizaje significativo para los adultos mayores que son oyentes de esta emisora.

1 El presente artículo es producto de la tesis para optar al grado de Maestría en comunicación educativa "Propuesta para mejorar los procesos educativos con los adultos mayores a través de la radio virtual comunitaria. Caso: Comuna 11 del municipio de Medellín, Antioquia" realizada por Juan Manuel Montoya y Marlon Echavarría Rodríguez. Universidad Tecnológica de Pereira.

2 Juan Manuel Montoya. Comunicador audiovisual, candidato a Magister en Comunicación Educativa. Jmontoyaagudelo@gmail.com

3 Marlon Echavarría Rodríguez. Productor de televisión, Comunicador social y periodista, Candidato a Magister en Comunicación Educativa. 
Palabras Clave: Emisora virtual, Educación Radiofónica, Radio, Enseñanza, Aprendizaje, Adulto Mayor

\section{Abstract}

A globalized world where the use of information technologies and communication has led to the creation, construction and consolidation of new proposals for teaching and learning. Mass media who has been forced to transform and evolve, to avoid becoming extinct in the past.

For this reason, from the interconnectivity offered by virtuality, and complexity that have digital immigrants in the use of technology, there is the proposal to analyze virtual community radio as educational, participatory and communicative process and transformation older adults in the commune 11 in Medellin.

The search for characteristic elements in the community and benefited of the community radio project, allowed to develop a qualitative research where through the questionnaire, data analysis and semi-structured interviews were conducted research "proposal to improve educational processes with older adults through community virtual radio. Case: Commune 11 of the city of Medellin, Antioquia" (Montoya \& Echevarria Rodriguez, 2015)

This process allowed to determine that from the creation of audience, follows a social transformation, since the content that is broadcast in the programs of $\mathrm{La}$ Once Radio generates significant learning for older adults who are this radio listeners.

Key Words: Virtual broadcasting station, Radio education, Radio, Teaching, Learning, Older adult

\section{Introducción}

El impacto social que generan los medios masivos de comunicación ha sido ampliamente estudiado (Arteaga \& Et Al, 2003) (Dean T. \& G. Mcnany, 1983) (Martínez , Pérez Frías, \& Solano, 2014) y diversas líneas han sido producto de éstos estudios, entre ellas, el interés por el componente educativo que desde allí se trasmita (Brennan , 2015) (Dean T. \& G. Mcnany, 1983). Sumado a éstas investigaciones, la globalización presenta un nuevo medio para la difusión de información y, de la misma manera, la producción de diversos contenidos enfocados en grupos poblacionales específicos. La virtualidad se convierte entonces en otro medio para generar impacto social (Jimenez Gil, 2014) (Martínez Pandini, 2014).

En la actualidad, los medios se han transformado o adaptado tanto a las necesidades y gustos de las personas como a los sistemas económicos que los gobiernan, "el poder de los medios puede variar con el tiempo" (McQuail, 2000) y a través de estos conocemos otro tipo de realidades y de experiencias; nuevos alcances y posibilidades que poco o nada se conocían (Gómez, 2015).

En Colombia, se ha realizado un universo de investigaciones sobre instituciones comunicativas comunitarias (Rodero, Vázquez, Larrea, \& Mas, 2014), es decir, sobreperiódicos, televisióny radioal interior de un sector específico de las ciudades con unos límites territoriales específicos (Alba Maldonado, 2015) (Alzate, 2011) (Arnedo Redondo, Hernández Guzmán, \& Jurado Vargas, 2014) Sin embargo, el contexto de cada una de ésta poblaciones y de las acciones que allí se realicen a partir de éstos medios comunitarios justifica la importancia de estudios enfocados en sus aristas y las múltiples intenciones de las 
emisoras virtuales (Bohada Rueda, 2015) (Lizarazo Castro, 2015).

Por tal razón, un estudio sobre los procesos educativos de enseñanza-aprendizaje con los adultos mayores a través de la radio virtual comunitaria abre el espectro investigativo (Szyszko, Neri, \& Cataldi, 2010), dado que se suman, además de la indagación por las instituciones comunicativas comunitarias, la utilización de la virtualidad enfocada a los adultos mayores de la comuna 11 del municipio de Medellín que deben ser catalogados como inmigrantes digitales (Prensky, 2001).

Por lo mencionado anteriormente, la probabilidad de encontrar en la radio virtual comunitaria, a partir de La Once Radio, un proceso con capacidad educativa de enseñanza-aprendizaje, en donde su contenido se construye de forma participativa y tiene como fin primordial el de comunicar el acontecer de su sector, termine por generar una transformación en la cosmovisión y la cotidianidad de su público objetivo.

La Once Radio es una emisora virtual creada en la comuna 11 que tiene como misión:

"Ser un espacio de comunicación para los 15 barrios que conforman la comuna, en la construcción de una sociedad con sentido de pertenencia, de participación ciudadana, con la producción de contenidos que reflejan la cotidianidad de la comuna y además que permitan valorar las particularidades y reconocer la importancia de la construcción de la historia de cada uno de sus habitantes" (Tomada de http:// laonceradio.fm/)

Desde su creación la emisora se planteó como un espacio comunicativo de la comunidad para la comunidad, la información de los eventos, actividades, procesos, problemáticas y proyectos se han informado por este medio desde el año 2011, permitiendo a toda la comunidad, ciudad, país y el mundo conocer lo que se hace desde los 15 barrios que conforman la comuna y lo que sus habitantes y líderes desarrollan para mejorar los procesos de convivencia, participación ciudadana, comunicación y educación.

En la búsqueda por la explicación y compresión de la posible transformación que genera la radio virtual comunitaria, fue elegido el paradigma históricohermenéutico en la medida en que permite entender al otro como un sujeto que puede ser analizado, explicado y descrito. Así mismo, con las herramientas de la investigación cualitativa (la entrevista semiestructurada, el cuestionario, la encuesta y el análisis documental) se indagó el equipo que constituye La Once Radio y a sus oyentes adultos mayores.

El proceso investigativo se inició en el mes de marzo del 2015, con el proceso de recolección de información que terminaría porlegitimarlos hallazgosy las conclusiones que de la investigación pudiesen derivar; tales como la conexidad de La Once Radio con su público objetivo; la relevancia de este hallazgo, por ejemplo, motivó la indagación por las acciones que permiten ese tipo de relación y que ayudan a mejorar los procesos de enseñanza-aprendizaje. Siguiendo este interés, se presentan las razones; entre ellas, que la conexidad se da por la generación de contenido construido de forma participativa, los talleres de capacitación en TIC's, la participación de los oyentes en la emisora, la finalidad de comunicar el acontecer de su sector y la producción de información a partir de los intereses de los oyentes. 


\section{Materiales y métodos}

Indagar por la cosmovisión y el impacto de La Once Radio en los adultos mayores de la comuna 11 de Medellín precisa de una investigación cualitativa que pueda describir la adaptación de éstos a un medio de comunicación virtual $\mathrm{y}$, además, la utilización que los adultos mayores hacen de los conocimientos allí transmitidos; en otras palabras, comprender y analizar la magnitud de las acciones educativas de un medio de comunicación virtual puesto al servicio de los ciudadanos adultos mayores.

Para efectos de la investigación, la importancia de generar participación con la comunidad a estudiar, a través de instrumentos de inclusión como lo son el análisis documental, la entrevista semiestructurada, y un cuestionario, permitió analizar el alcance que tiene un medio de comunicación como lo es la radio virtual comunitaria en un proceso de enseñanza - aprendizaje con los adultos mayores de la comuna 11 del municipio de Medellín.

La realización de entrevistas semiestructuradas al equipo que conforma La Once Radio, entre ellos su coordinadora y quienes dirigen los programas más escuchados por los adultos mayores. A saber, "El taller del idioma", "Deporte y convivencia" y "El Escaparate". De igual forma, se realizaron 4 entrevistas semiestructuradas a adultos mayores que se declararon fiel oyentes de la emisora con la intención de establecer cómo a través de la radio virtual se da un proceso de enseñanza - aprendizaje, además de conocer las ventajas y desventajas de éste medio. Por otro lado, fue importante la puesta en marcha de la estrategia, ya que permitió saber la opinión que tienen los adultos mayores de esta comuna entorno a su vinculación, a su edad y a un proceso formativo de carácter virtual.
Las personas mayores de 50 años que habitan la comuna 11 de Medellín es el universo de la encuesta realizada. Sin embargo, el marco muestral fue enfocado en aquellos adultos mayores que tenían un previo proceso de vinculación como oyentes de La Once Radio. El tamaño de la muestra fue de un total de 100 adultos mayores que se constituían como oyentes de la emisora virtual comunitaria de la comuna 11 de Medellín.

Dadas las dificultades para acceder a los oyentes a partir de los registros web, el cuestionario fue realizado en los lugares frecuentados por los adultos mayores de la localidad. Los parques, las cafeterías y las aceras fueron los lugares en donde se abarcó el tamaño muestral de la investigación.

Posteriormente y luego de aplicar los instrumentos de recolección, la información fue organizada de acuerdo a cada instrumento y objetivo. Es decir la transcripción de cada una de las entrevistas semiestructuradas llevadas a cabo tanto a los adultos mayores como a los periodistas - realizadores. Por su parte, el cuestionario se organizó de acuerdo a cada pregunta, lo que significó determinar la cantidad de personas que se sentían identificadas con las respuestas allí brindadas, esto hace una comprensión del estudio, lo que facilitó la sistematización de la información.

Finalmente, se realizó una matriz de análisis documental, presentada en el gráfico 1.1, donde se plantea la definición de unos anclajes que permitan delimitar más la información después de la descripción, identificando puntos claves de los datos que se recopilaron; luego vienen los conceptos que son un modo de presentar, de manera más precisa en la matriz, el análisis que resulta de descifrar el texto. Estos datos empíricos que luego de procesados se convierten en información crucial para descubrir los aspectos más relevantes de 
la investigación, desarrollar los objetivos, alcanzar los productos o resultados esperados, agotar el proceso metodológico y, lo más importante, producir un nuevo conocimiento.

\begin{tabular}{|l|l|l|l|}
\hline \multicolumn{5}{|c|}{ Matriz de anélisis documental } \\
\hline Descripción & Anclajes & Conceptos & Categorias emergentes \\
\hline Radio Educativa & & & \\
\hline Educación Radiofónica & & & \\
\hline Estudiante Adulto & & & \\
\hline Comunicación Educativa & & & \\
\hline Enseñanza Aprendizaje & & & \\
\hline
\end{tabular}

Gráfico 1.1 Matriz de análisis documental

\section{Resultados}

La búsqueda por encontrar en la radio virtual comunitaria un proceso con capacidad educativa (Manduley, 2015) de enseñanza-aprendizaje, que termine por generar una transformación en la cosmovisión y la cotidianidad de su público objetivo, se acerca a los resultados obtenidos por La Once Radio dado el impacto que tiene por la comprensión de su medio como una alternativa de aprendizaje (desde el concepto de la información, enseñanza y aprendizaje), así mismo por su capacidad de posibilitar espacios de encuentro, de diálogo, de discusión y análisis de los contenidos emitidos por ella y el desarrollo de métodos de información para la educación y el aprendizaje.

La conexidad de La Once Radio con su público objetivo, generado por el contenido que se construye de forma participativa, los talleres de capacitación en TIC's, la participación de los oyentes en la emisora, la finalidad de comunicar el acontecer de su sector, y la producción de información a partir de los intereses de los oyentes, permite una correlación permanente entre el oyente, los periodistas-realizadores y el medio de difusión; tal y como se demuestra en el gráfico 1.2. De ésta forma se genera el sentido de la educación puesto que
"Enseñar no es transferir conocimiento, sino crear las posibilidades para su producción o construcción. Quien enseña, aprende al enseñar y quien enseña aprende al aprender" (Freire, 1996, pág. 47)

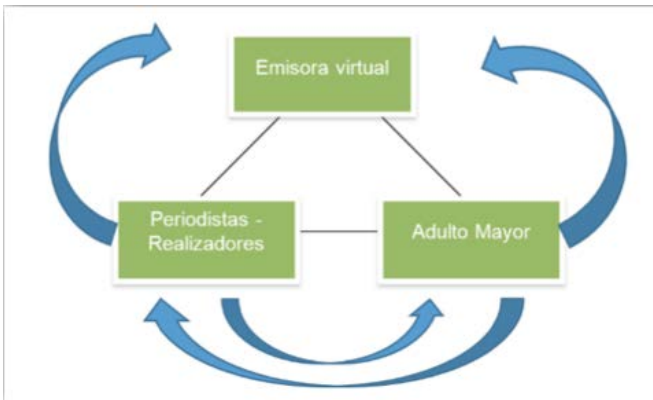

Gráfico 1.2. Conexidad entre emisora - oyente radio virtual

Consecuentemente, La Once Radio al interior de la comuna 11 y en Medellín se ha convertido en un medio importante para la ciudad, puesto que se ha transformado en el medio o vehículo de difusión para hacer llegar a las comunidades el discurso de las políticas públicas gubernamentales. Cobrando así importancia por mantener al tanto de los asuntos públicos de interés general a sus oyentes. La comunicación de los asuntos públicos crea un puente importante para fidelizar audiencia (Carrillo \& López, 2015).

Por otro lado, emergen en la investigación diferentes expresiones y definiciones que se encontraron en el proceso de consolidar la información obtenida con los instrumentos aplicados. Estos conceptos que vemos en la gráfica 1.3, complementan la descripción previa que se había realizado. Los conceptos de Emisora virtual, aprendizaje radiofónico y educación virtual enmarcan las definiciones pre establecidas en la investigación. 


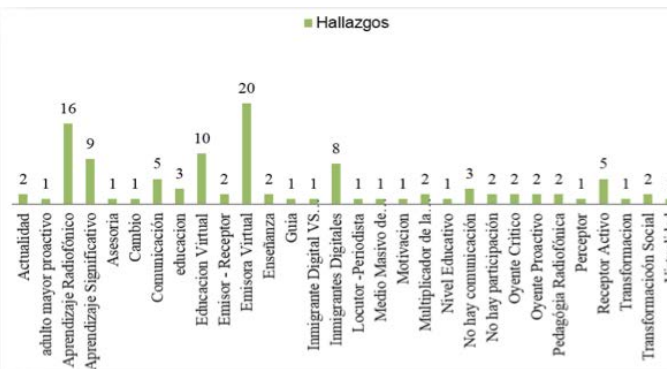

Gráfica 1.3. Conceptos hallazgo

La accesibilidad al medio virtual se presenta como uno de los principales obstáculos para la conexidad mencionada anteriormente. Sin embargo, sumado a los talleres dictados por La Once Radio que resultan ser efectivos, éste límite es superado cuando el núcleo familiar del oyente intercede posibilitando el acceso a la página e igualmente el adulto mayor decide su condición de inmigrante digital y opta por aprender a conectarse desde otros aparatos móviles. En el curso de la indagación fue posible evidenciar la forma en que se solventan éstos límites; los resultados se presentaran en la gráfica 1.4 y 1.5 respectivamente.

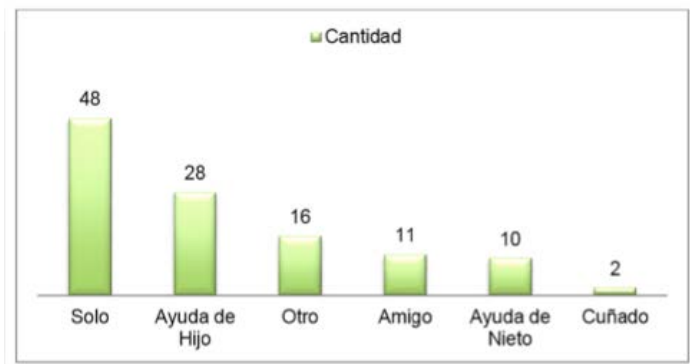

Gráfica 1.4 Núcleo familiar para acceso a la emisora

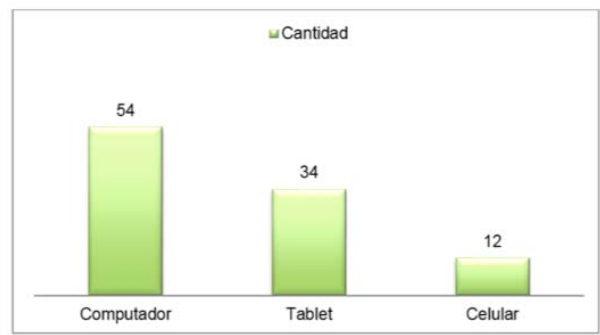

Gráfica 1.5 Medio utilizado para acceder
Al comprender la forma en que se da la conexión entre la emisora y el oyente en el proceso de enseñanza - aprendizaje es posible realizar conjeturas deductivas. La construcción de una propuesta para mejorar los procesos educativos con los adultos mayores a través de la radio virtual comunitaria es posible si se entiende que las dificultades más apremiantes se pueden resolver por medio de la comunicación constante entre uno y otro.

\section{Discusión de resultados}

Los medios masivos de comunicación entendidos como una herramienta fundamental para poner de acuerdo a los procesos de enseñanza y aprendizaje (Rodero Antón, 1997, pág. 5) conllevan un proceso de adaptación que, en el caso de La Once Radio, inicia con algo trascendental y fundamental en los medios masivos comunitarios: la creación de su propias audiencias. En la gráfica 1.6 se describe el proceso por medio de cual se fideliza la audiencia de la once radio y que permite no sólo la permanencia de ésta al aire, sino que viabiliza la transformación de su público objetivo por medio de la enseñanza - aprendizaje.

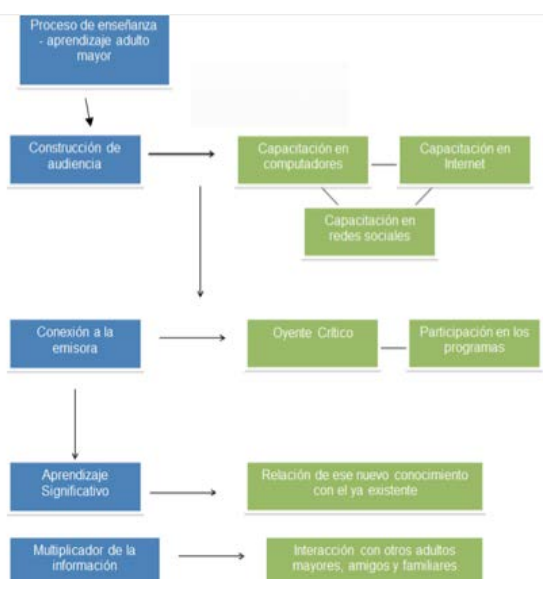

Gráfica 1.6 Proceso enseñanza - aprendizaje de la Once Radio. 
Como se ve reflejado en la figura anterior el proceso de enseñanza - aprendizaje inicia con la construcción de audiencias, este es uno de los hallazgos importantes de este proceso investigativo, por lo que es, este el punto de partida de este camino, ya que es allí donde se cimientan las bases de este proceso.

En este sentido Teresita Pérez la coordinadora de la emisora La Once Radio expresa lo siguiente:

“Acá hemos hecho alfabetizaciones en informática, pues el único medio de información que tiene la comuna es la once radio... entonces nosotros hemos hecho mucho por crear audiencia, anualmente hacemos 10 talleres cada uno de 20 horas para grupos de 16 personas, los talleres son de tres niveles el primer nivel es para quien nunca ha usado el computador, entonces tiene que ir allá a saber que es el mouse, como lo prende, el segundo nivel es para quien no sabe cómo usar la internet, y la emisora... y el tercer nivel es para quien no tiene correo electrónico ni Facebook, allí se lo creamos... pero eso si a mí lo que me interesa es que queden duchos en navegar La Once Radio, por lo que nos interesa es que entren a la emisora, se enteren que está sucediendo en la comuna". (Entrevista semiestructurada realizada a Teresita Pérez, 7 de abril de 2015).

La construcción de audiencias es el camino para consolidar a futuro los oyentes de la emisora, allí se lleva a cabo una construcción de nuevos saberes, puesto que los adultos mayores son conocidos como inmigrantes digitales, es decir, que el contacto que han tenido o pueden tener con los dispositivos digitales es limitado. Este nuevo conocimiento parte entonces con la enseñanza y aprendizaje del computador como herramienta o medio de comunicación, de allí luego se pasa a la navegación en internet en donde ésta

"No es solamente una herramienta de comunicación y de búsqueda, procesamiento y transmisión de la información que ofrece unas prestaciones extraordinarias; internet conforma además un nuevo y complejo espacio global para la acción social, y por extensión, para el aprendizaje y la acción educativa". (Coll \& Monereo, 2008)

Posterior a conocer como es el mundo de la navegación en la web, sigue la participación, es decir, se transforma el receptor en perceptor. Es en este punto donde las redes sociales como Facebook cumplen un papel fundamental, ya que es a partir de éstas que los oyentes pueden interactuar con la emisora. Tal como lo indica Esteban Zárate, adulto mayor y habitante de la comuna 11 de Medellín, quien expresa que:

"El actual momento de las comunicaciones es a través de los medios virtuales, ya los viejitos que predominan en esta comuna o adultos mayores por respeto, cierto, están incursionando en los medios virtuales, casi peleando con los hijos para el control del computador. Hoy la radio virtual es la forma más masiva de crear redes sociales y de aprender de procesos, de eso yo estoy absolutamente convencido". (Entrevista semiestructurada realizada a Esteban Zárate, 22 de mayo de 2015)

La entrevista semiestructurada como instrumento de recolección de la información, también permitió establecer que sí se aprende a través de la radio virtual 
comunitaria, tal y como lo indica Margarita María Gómez:

"Si he aprendido porque cuando uno entra a cualquier espacio a cualquier emisora y tú le pones el cuidado pues donde esté recibiendo alguna información y tú le pones el cuidado, si de verdad desde tu interior quieres realmente aprender pues lo haces". (Entrevista semiestructurada realizada a Margarita María Gómez, 21 de mayo de 2015).

Así mismo, Emma Rodero Antón en el proceso de aprendizaje por medio de la radio da a conocer que este medio masivo de comunicación puede hacer "decidir por sí mismas, a aprender por cuenta propia, a comportarselibre,felizyresponsablemente" (Rodero Antón, 1997, pág. 5) Conforme a la pregunta por si ¿Aprendiste al escuchar la emisora? mostrada en la gráfica 1.7 , se puede establecer que más de la mitad de las personas $(64 \%)$ que sirvieron como muestra de conveniencia, sí aprenden al momento de escuchar La Once Radio.

El otro 36\% restante se divide en los siguientes porcentajes: el 17\% de ellos da a conocer que no realiza esta acción por medio de este medio, el 14\% dice que a veces, mientras que el $5 \%$ dice que depende del tema, el cual se puede expresar que está intrínsecamente ligado al gusto personal.

El ideal se convierte entonces en que se escuchen los contenidos emitidos por la emisora, que se ponga en práctica lo escuchado, que se conviertan en perceptores no acríticos de los contenidos, en que la información que reciban se perciba de forma crítica; que los contenidos que se reciban entren en juego con todos sus imaginarios, ideas, experiencias y que se ponga en juego para el ejercicio diario de cual sea el rol que desempeñen.

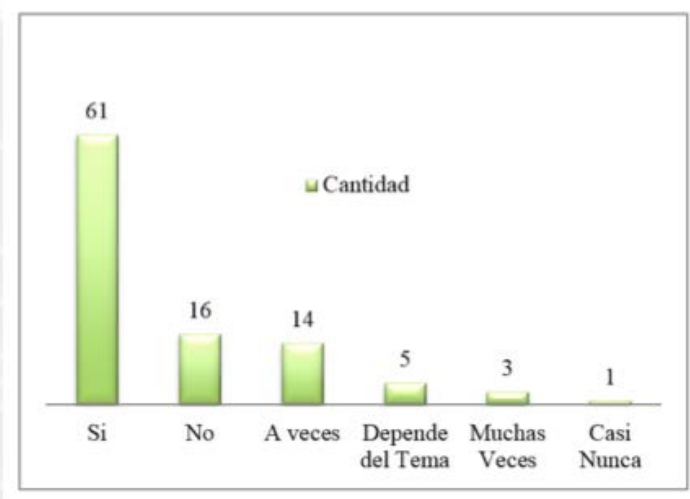

Gráfica 1.7 Respuestas a la pregunta ¿Aprendiste al escuchar la emisora?

Así mismo, el aprendizaje que se adquiere a través de este medio no se queda solamente en ellos, se multiplica, por lo que los adultos mayores se vuelven en replicadores de la información en tanto es posible cuestionarse los planteamientos emitidos por la emisora al tiempo que se le escucha. La gráfica 1.8 que se ilustrará a continuación permite conocer más del 90\% de los adultos mayores expresa que sí estudia al momento de escuchar la emisora. Unos los hacen muchas veces, sin embargo, otros expresan que pueden realizarlo en una frecuencia muy relativa, es decir que ésta varía entre a veces o podría ser; sin embargo, la posibilidad de encontrar allí opiniones valiosas también motiva la atención para con los contenidos.

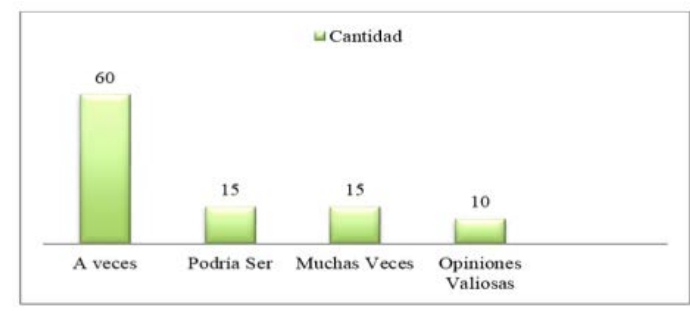

Gráfica 1.8 Respuesta a la pregunta ¿estudias desde la radio? 
Al conocer que las temáticas que más aprenden los adultos mayores se debe a su interés por temas específicos, lo que ayuda a la emisora en su proceso de construcción y reconstrucción permanente como medio de comunicación comunitario, es importante conocer lo expresado por Sarramona cuando afirma que

"La funcionalidad se garantiza cuando los contenidos están ligados a la realidad que envuelve a los adultos participantes en el programa. Así pues, los contenidos no pueden ser fruto de la sola especulación de los planificadores del programa, sino que han de surgir del propio grupo destinatario. (Sarramona, 1982)

Por tal razón, toma importancia el conocimiento obtenido al cuestionar por las temáticas que más interesan y en la cual aprenden más los adultos mayores. Los resultados obtenidos y que son especificados en la gráfica 1.9, demuestran que los programas entorno a la cultura $(41 \%)$, la salud $(27 \%)$, la política $(27 \%)$, el deporte $(12 \%)$, la nutrición $(12 \%)$, y la ortografía $(7 \%)$, son los que más alimentan ese proceso de enseñanza y aprendizaje que se da en la emisora virtual comunitaria La Once Radio.

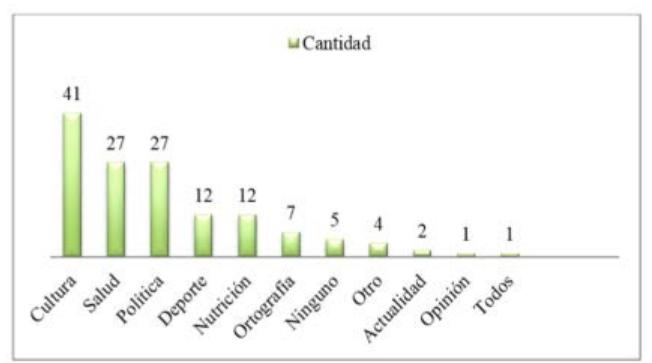

Gráfica 1.9 Temáticas que interesan al público objetivo

Con respecto a la transformación social de La Once Radio en la vida de los adultos mayores oyentes de la emisora; ésta se da a partir de la participación, el pensamiento y la forma de relacionarse, como se ilustra en la gráfica 1.10.

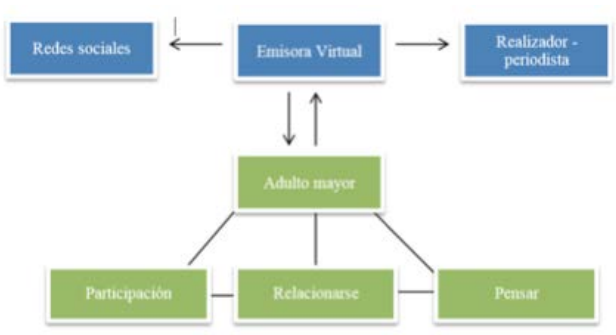

Gráfica 1.10 Transformación social por medio de la participación, la forma de relacionarse y el pensamiento

Es posible afirmar que la emisora como punto de encuentro, comunicación y socialización de la información en la comuna 11 de Medellín, ha posibilitado que en los adultos mayores que la escuchan se generen transformaciones, ya sea de índole interno o externo al ser humano. La participación ha sido uno de los elementos fundamentales en la transformación de estos adultos mayores, la emisora se ha convertido en el medio de aprendizaje e información. Esta herramienta ha posibilitado que exista entre ellos mayor participación en discusiones y conversaciones que desarrollan con sus familias y amigos como lo indica Margarita María Gómez al afirmar que:
"El poder acceder a ese tipo de información es importantísimo, por que mira uno se nutre, uno se siente, cambia la actitud cambia, la forma de pensar y la visión al mundo es otra. Para mí eso es enriquecedor, básico y fundamental. La formación es un alimento" (Entrevista semiestructurada realizada a Margarita María Gómez, 21 de mayo de 2015).

Ángela Montoya, oyente de La Once Radio y activista de la comuna, se refiere a la emisora como ese medio que ha forjado en 
ella cambios drásticos para su vida. Con su testimonio da validez en donde la edad no es una barrera o un limitante para aprender, cambiar y mejorar:

"Yo era más tímida para hablar, pero ya hablo más, ahora soy más analítica me gusta intervenir más, es decir, espero que se abra el debate para poder analizar las cosas y conocer los puntos de vista de los demás y aportar en el momento ideal. La emisora me ha brindado, me ha facilitado el aportar esos conocimientos". (Entrevista semiestructurada realizada a Ángela Montoya, 8 de mayo de 2015).

Los habitantes mayores de la comuna 11 de la ciudad de Medellín, apropian el medio de comunicación a sus quehaceres diarios, la asumen como una alternativa de información y comunicación local, que se convierte en el sentir de todos, que los conoce y conoce sus gustos, sus afinidades, sus problemáticas y en cierta medida trata de resolverlas de la mejor forma. El adulto mayor de los barrios que conforman la comuna 11 identifica la emisora como un medio para aprender.

\section{Conclusiones}

La construcción de una propuesta para mejorar los procesos educativos con los adultos mayores a través de la radio virtual comunitaria debe estar mediada por la investigación cualitativa; el reconocimiento de la subjetividad del individuo para entender su contexto y, a partir de su percepción, generar datos empíricos que permitan comprender su realidad y a partir de ella planear el mejoramiento de sus condiciones.

En concordancia con lo anterior, un estudio a partir de la investigación cualitativa permite que los adultos mayores de la comuna 11 de Medellín puedan reconocer en la radio virtual comunitaria un espacio de interés y, así mismo, la investigación en curso pueda alcanzar el cumplimiento de sus objetivos y aportar a la comunidad académica un nuevo conocimiento.

La radio virtual comunitaria en el contexto de la comuna 11 de Medellín no sólo sortea problemas con respecto a las dinámicas afines al mercado, sino que, teniendo como público objetivo los adultos mayores, se enfrenta a las dificultades presentadas por los mismos para adaptarse a las nuevas tecnologías. Acudir a herramientas que posibiliten el contacto entre el público objetivo y la emisora es el primer medio al cual se debe la comunicación.

En este sentido, los talleres de capacitación en la web y en los recursos tecnológicos desde donde puede ser escuchada la emisora, inician el proceso de aprendizaje y adaptación de los inmigrantes digitales; puesto que incentiva espacios de encuentro en donde el dialogo, los debates y el análisis de los contenidos terminan por consolidar la aprehensión de los conocimientos emitidos por la emisora, la cual cuenta ya con una franja fiel de oyentes.

Igualmente, el quehacer cotidiano presenta oportunidades para reconocer y aprehender nuevos saberes. Sin embargo, la no difusión o no repetición de estos conocimientos termina por obviar su utilidad y encuentran prontamente su olvido. La Once Radio, al tener como público objetivo los adultos mayores, su contexto y sus intereses, reconoce en la difusión de lo allí tratado el mecanismo propicio para mantener su audiencia y permanecer al aire en el contexto de la globalización.

Sumado a esto, ofrecer contenidos que puedan ser tomados como propios por los adultos mayores permite un proceso 
de aprendizaje que posteriormente será un proceso de transformación. En la medida en que los contenidos emitidos son interiorizados y difundidos por los radio escuchas, su cosmología y la forma en que entienden los acontecimientos diarios cambian y más si éstos conocimientos se hacen merecedores de debates colectivos que terminan por reforzar una idea colectiva de un fenómeno específico.

Por tal razón, la conexión y los lazos que se generan entre La Once Radio y los adultos mayores de la comuna 11 de Medellín se convierten en un proceso de identificación y aprendizaje no direccionado. La relación existente entre ambas partes produce una sinergia ante la posibilidad de educarse desde la comunicación y el interés de educarse para comunicar; puesto que la comunicación es un proceso transversal entre la necesidad de comunicarse para compartir cosmologías y el del desarrollo de habilidades para relacionarse.

Es posible afirmar, a partir de las categorías emergentes evidenciadas anteriormente, que la posibilidad de integrar la web por medio de una emisora virtual y un componente educativo puede generar un proceso de transformación que avanzará de forma eficaz, efectiva y eficientemente. La inmediatez y la accesibilidad de la web, sumado a la aprehensión y difusión de los conocimientos que se adquieren, generan un efecto de "bola de nieve", es decir, se reproduce de vos a vos y cada vez con más impacto.

A ésta técnica se debe el sostenimiento en el tiempo de La Once Radio, pues su sentido comunitario, basado en el reconocimiento de su población objetiva, produce un ambiente propicio para que sea escuchada. Además, las actividades que realiza en capacitación a los adultos mayores en nuevas tecnologías e ingreso a la web permite crean un vínculo amistoso y las condiciones necesarias para que sus oyentes puedan permanecer conectados con la radio que sienten propia.

\section{Referencia Bibliográfica}

Alba, J. M. y Garcia, A. C. (2015). Inclusión educativa de la población rural en el departamento de la Boyacá-Colombia a través de la virtualidad en el modelo pedagógico de la Universidad Nacional Abierta y a Distancia UNAD. Colombia: Universidad Nacional Abierta y a Distancia UNAD.

Alzate,J.R.(2011). "Porfinhayradiocomunitaria en Medellín. En Medellín cultura: una sola ciudad". Consultado el 8 de junio de 2015, http://www.medellincultura.gov.co/laescena/ Paginas/LE_radiocomunitariamedellin. aspx\#IDComment568797462

Arnedo, B. L; Hernández, D. y Jurado, J. J. (2014). El laberinto de las radios comunitarias en Bolívar: entre la subsistencia y el aporte social al desarrollo comunitario. Palobra, No 14, pp. 238-256.

Arteaga, C. et al. (Diciembre de 2003). "Un paso por al historia de la radio". consultado el 07 de mayo de 2015. En: Razón y Palabra, http://razonypalabra.org.mx/anteriores/n36/ carteaga.html

Bohada, X. A. (2012). Fortalecimiento de las comunicaciones internas y externas de la facultad de comunicación social - periodismo de la UPB Bucaramanga, haciendo uso de medios virtuales y herramientes tecnológicas. Trabajo de grado. Bucaramanga: Universidad Pontificia Bolivariana.

Ortiz Brennan, B. (1974). Análisis comparativo sobre las ventajas y limitaciones de los medios de comunicación para la educación. En: Chasqui Revista Latinoamericana de Comunicación, 1(6), 11-32.

Ortega, J. y Rendòn, L. (2015). Aprendizaje colaborativo mediante redes sociales y radio comunitaria web 2.0. En: Revista Lasallista de Investigación, 12(2). 
Coll, C., y Monereo, C. (2008). Educación y aprendizaje en el siglo XXI: nuevas herramientas, nuevos escenarios, nueva finalidades. En: C. Coll, \& C. Monereo, Psicología de la educación virtual (págs. 1953). Madrid: Morata.

Dean T., J., \& G. Mcnany, E. (1983). La radio al servicio de la educación y el desarrollo. En: N. r. Medias, La técnica de la enseñanza. España: Servicio de publicaciones del Ministerio de educación y ciencia.

Freire, P. (1997). Pedagogía de la autonomía. México: Siglo XXI.

Jimenez Gil, J. (2014). Las representaciones sociales y los medios masivos de comunicación: una vía de encuentro entre ambos conceptos. Monografía. Pereira: Universidad Católica de Pereira.

Lizarazo Castro, C. (2015). Diseño de una emisora en la web para difundir contenido lúdico, responsable y cultural en los bogotanos. Tesis de grado. Universidad de la Sabana.

Manduley, J. (1976). El foro de radio rural como una estrategia para educar adultos para el desarrollo comunitario. Chasqui Revista Latinoamericana de Comunicación, 1(13), 5776.

Martínez, M;Pérez, W. y Solano, D. (2011). Impacto de los medios masivos de comunicación en la dinámica familiar. Cultura, Educación, Sociedad - CES, 2(1), 111-118.

Martínez Pandini, G. (2006). El impacto de la televisión en la comunicación política moderna. Signos Universitarios, 25(1), 69-88.

McQuail, D. (2000). Introducción a la teoría de la comunicación de masas. Barcelona: Paidós.

Montoya, J., \& Echavarría Rodríguez, M. (2015). Propuesta para mejorar los procesos educativos con los adultos mayores a través de la radio virtual comunitaria. Caso: Comuna 11 del municipio de Medellín, Antioquia. Tesis de Maestría. Colombia: Universidad Tecnológica de Pereira.

Prensky, M. (2001). Nativos digitales, inmigrantes digitales. En: On the Horizon ((MCB University Press), 9(6), pp 1-7.
Rodero Antón, E. (1997). La radio educativa. Salamanca: Universidad Pontificia de Salamanca.

Rodero, E., Vázquez, M., Larrea, O., \& Mas, L. (2014). Sonidos que educan entreteniendo. La radio en la educación 2.0. En: J. R. Terceño, Creaciones audiovisuales actuales (pág. 411). Madrid: Asociación Cultural y Cientifica Iberoamericana - ACCI.

Sarramona, J. (1982). Principios que justifican la metodología no formal en la educación de adultos. Educar(1), 49-60.

Szyszko, N; Neri, C. y Cataldi, Z. (2010). $\mathrm{La}$ radio en la escuela media como agente participativo. Tesis de grado. Buenos Aires: Quaderns digitals: En: Revista de nuevas tecnologías y sociedad. 